\title{
Early interceptive treatment management
}

\author{
M. Limme ${ }^{1}$, A Bruwier ${ }^{2}$
}

1 Professor of dentofacial orthopedics and of pediatric dentistry, University of Liège, Belgium. Department head of dentofacial orthopedics and pediatric dentistry at University Hospital of Liège

2 Senior orthodontist, clinical supervisor of the dentofacial orthopedics and pediatric dentistry at the University Hospital of Liège Institute of dentistry - L. Brull Policlinic - Quai G. Kurth 45, 4020 Liège - Belgium

\begin{abstract}
This article offers a series of justifications for early interceptive treatment as opposed to later treatment that might necessarily involve the extraction of permanent teeth. The biological responses of the membranous sutures, of the periosteum, of the periodontal ligaments, and of the condylar cartilages but also of the potential for growth in stature, well before the pre-pubertal growth spurt and the administration of effective treatments, are also reasons that support an early phase of treatment, in the early mixed dentition, even in the primary dentition!

Some early orthodontic or orthopedic treatments and their appliances such as buccal screens, myofunctional trainers, occlusal splints or sectional expansion plates and other maxillary expansion and protraction appliances are useful for correcting deformities or severe under-developments that are already in place.

But myofunctional re-education, when carried out according to a structured and prioritized program, is perhaps the most important because these etiopathogenic treatments used to both suppress the causes of the deformations and to restore balanced function, make it possible to re-establish a normal growth pattern and perhaps consequently, to avoid the need for extraction of permanent teeth.
\end{abstract}

\section{KEY WORDS}

Interception, early treatment, non-extraction treatment, speech therapist/pathologist, myofunctional re-education

\section{INTRODUCTION}

Early orthodontic treatment is recommended by some and conversely avoided by others, who prefer to start treatment at a later time, often in the complete dentition. A great number of practitioners support the latter position, notably because of the

Address for correspondence:

Michel Limme

Institut de Dentisterie Policlinique L. Brull

Quai G. Kurth 45, 4020 Liège

Belgique

michel.limme@ulg.ac.be

Article received: 29-10-2013.

Article available at nttp://Www.jdao-journal.org or nttp://dx.do 
increasingly systematic use of multibracket techniques. These techniques have proven to be effective for a long time and many parents, who have heard about this procedure and are aware of it, can't help but be surprised when a practitioner suggests that their child begin treatment at a much younger age in the mixed dentition or even in the deciduous teeth.

When faced with a very young patient presenting with a developing or an already developed malocclusion, the orthodontist often says: "the child is too young," "we can't do anything right now", "we should wait for all the permanent teeth", "we should wait until the pre-pubertal growth spurt has started."

After hearing these arguments, that parents easily understand since they "make sense," many young children were often left untreated until the age of 12 .

We're forgetting that the young person sitting in front of us is in the growth phase and that, over the years, the expression of growth can be modulated, especially in regard to dysfunctions that have already led to the development of deformations at a young age. Since these causes were not detected or treated early, there is

\section{IS EARLY TREATMENT JUSTIFIED?}

It seems, in effect, more prudent to manage an anomaly as soon as it is first diagnosed. An interceptive approach such as this certainly seems to be a very attractive option. Nevertheless, we have every right to ask if it is truly realistic and if the efforts made can produce a significant improvement an even greater risk that existing dental malpositions and malocclusions will become worse over time. The severity of the problems can then be such that the extraction of permanent teeth with extended treatment time and more complicated treatment is frequently necessary. On the other hand, by waiting we lose the therapeutic opportunities that interventions and growth responses provide which are only possible when the growth potential is present. The possibility of obtaining these orthopedic responses is lost and in certain cases, orthognathic surgery can no longer be avoided.

If this is the case, we might ask, when faced with a young child presenting early signs of orthodontic problems, why is it that we adopt a passive attitude rather than trying to intercept them and restore morphological and functional harmony as soon as possible, by initiating early treatment that is simultaneously corrective and preventative.

From this perspective, orthodontics is probably the only discipline in the medical world where practitioners take the liberty of delaying treatment for several years, when confronted with a pathology!

in young children. In fact, we are all entitled to ask some questions:

- are the affected dento-alveolar and basal dental structures both likely to produce adaptive morphological responses after functional or mechanical therapeutic interventions, or, on the contrary, are we dealing 
with congenital anomalies that cannot be corrected in very young children? In other words, "is compensatory growth possible?";

- is the growth potential of a young child well before the pre-puberal growth spurt sufficient to allow for adequate quantitative responses at that age? In other words, "will the compensatory growth be sufficient?";

- If normalization is achieved at a young age, will it maintain itself or, on the contrary, lead to a relapse because it was obtained too soon before the complete eruption of the permanent teeth? In other words, "is the compensatory growth stable?";

- finally, can we really expect these very young children to be sufficiently cooperative and to accept that they have to wear orthodontic appliances?

Only a positive response to these four questions can justify the use of early treatment and, if this is the case, orthodontic appliances should be recommended on a much more regular basis.

Are the involved dento-alveolar and basal structures, themselves the products of adaptive morphological responses due to functional or mechanical therapeutic stresses, or, on the contrary, are we dealing with congenital anomalies that cannot be corrected in very young children? In other words, "is compensatory growth possible?"

The various bony parts of the craniofacial skeletal structure have a common mesenchymal yet distinctive origin beginning from the first stages of their embryological development in relation to the ossification process that is encountered.

- In the mesoblast of certain cephalic regions some kernels of cartilaginous cells appear that, by proliferating, lead to the formation, starting from the third month in utero, of the primordial chondrocranium. Close to the future cranial base, the cartilaginous pieces (ethmoid, pre-sphenoid, basisphenoid, and basi-occipital) will be deeply invaded by an endochondral ossification process during which they continue their peripheral growth, particularly at the synchodroses, bipolar zones of cartilaginous proliferation. Around 3 years of age, these synchondroses close and ossify, with the exception of the spheno-occipital synchondrosis which continues to grow until puberty (Fig 1).

- On its inferior face, the nasal capsule projects two extensions that give rise to inferior turbinates and a median extension, the important cartilage of the nasal septum, that remains active until the end of growth. Meckel's cartilage, that is also produced by this primordial cartilaginous matrix, is the rough outline of the future mandibular trajectory but it will disappear for the most part without undergoing endochondral ossification. Only its superior extremity will transform and give rise to one of the small bones of the middle ear, the malleus, together with the anterior ligaments of the malleus and the spheno-mandibular ligaments. Its anterior extremity will leave a few 


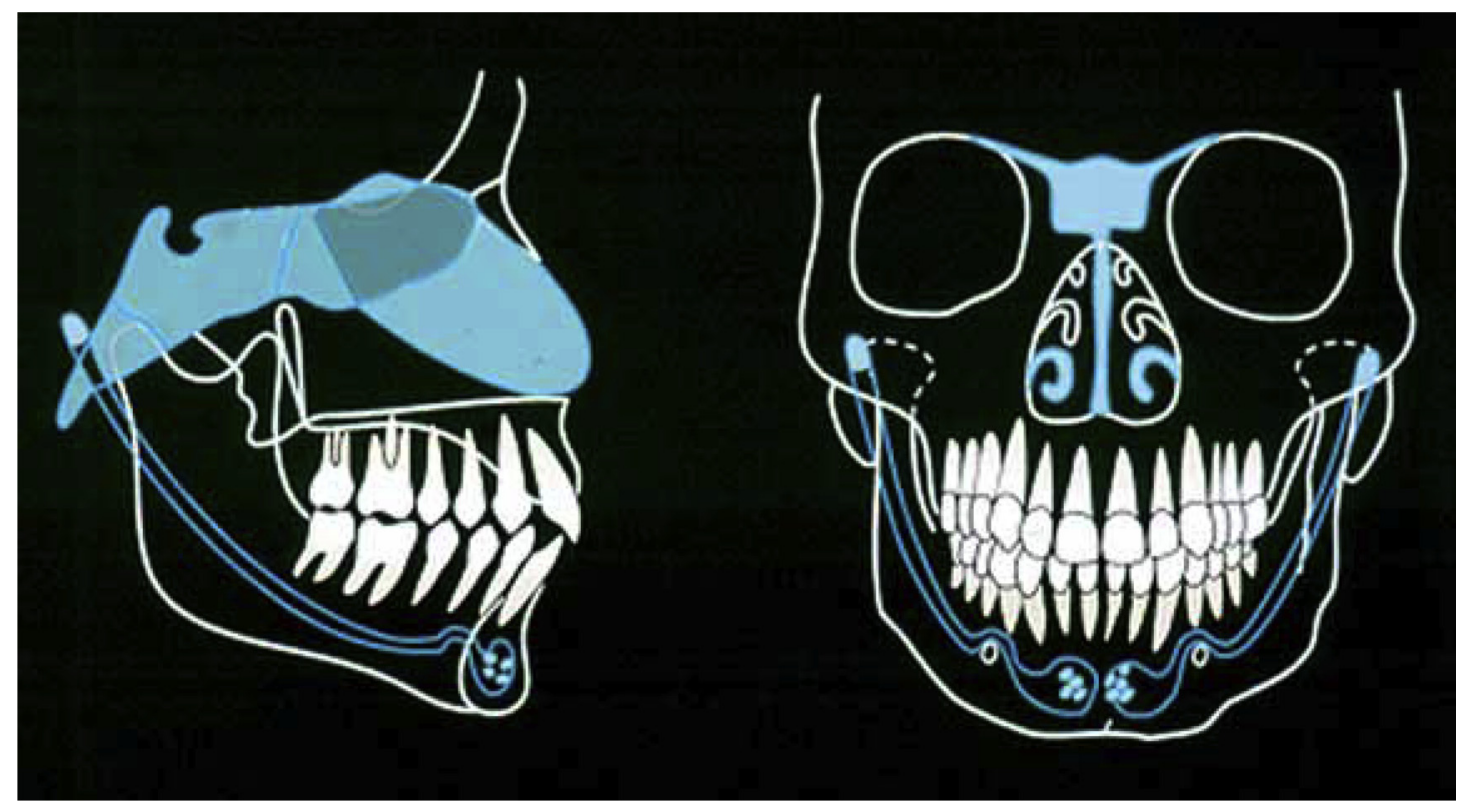

Figure 1

In blue, structures of cartilaginous origin that result from endochondral ossification: the cranial base and the synchondroses, the septal cartilage and the inferior turbinates, Meckel's cartilage and the chondriola symphysae.

cartilaginous islands that will ossify and become part of the symphysis (chondriola symphysae).

The cartilaginous structures present growth that is essentially predetermined, subject to genetics and certain epigenetic factors but independent of local factors. Primordial cartilage, or a growing synchondrosis when placed in an organic culture medium will continue to produce mitoses, as if there was a predetermined pattern and will respond positively to the addition of growth hormone. It therefore has an inherent growth potential that is barely influenced by mechanical environmental factors. In addition, the epiphyseal cartilages of our long bones are capable of proliferating in spite of the pressure conditions gravity exerts on them. Therefore, we can readily understand that these cartilages as well as the bony pieces that result from them are, on one hand, barely influenced by the functional environment but also, on the other hand, only slightly modified by the mechanics of orthopedic treatment at a young orthodontic age.

However, some studies, especially those led by M.J. Deshayes ${ }^{4}$, seem to show today that some functional and mechanical treatments of maxillary deformations performed very early, before the age of 6 years, can contribute to reshape, reposition or reorient some basicranial structures. The orientation or the atypical or asymmetrical position of these basicranial structures is the source of the abnormal arrangement of the facial structures

- Most of the bones of the face and the cranial vault have a different origin. Within the mesenchyme 
where most of the bones of the cranial vault and the face are formed, the cells differentiate immediately into osteoblasts that begin to secrete an osseous substance. Once the osseous spicules are formed, they proliferate in a radial fashion towards the periphery. The primary kernels resulting from this process of membranous ossification meet at some surfaces but leave in the interstitial spaces a band of connective tissue that displays on each side an osteogenic layer in contact with each one of the two growing osseous pieces: these are membranous sutures. These sutures are growth sites but not inherent growth centers. As J. Delaire states, these are "marvelous joints with automatic compensatory growth." For a suture to proliferate and produce osseous growth at its edges, it has to be subjected to tensile mechanical factors to then orient itself perpendicularly to the resultant of the direction of the tractions. The responses of the growth of the sutures are therefore dependent on environmental factors. When the environmental factors are not present, and in particular when the primary displacements of the osseous pieces induced by the motors of growth stops, the sutures, now put at rest, lead to the closure of the suture at variable ages based on their location. When isolated in an organotypic culture medium, a suture slows then stops growing. The addition of a growth hormone slightly increases the proliferation, but, deprived of environmental mechanical stimulation, it will not show any further adaptive growth.

However most of the bones of the middle third of the face (maxilla, palate, vomer, zygomatic bone . . .) have between them many sutural surfaces that are oriented in different planes that are indicative of the stimulations and responses of three dimensional growth present in this region.

In addition, the osteogenic sutural surfaces extend as an osteogenic membrane that makes up the internal layer of periosteum, which lines all of the osseous surfaces. It's the periosteum, in connection with the soft tissues of the facial envelope, that will progressively, by apposition and resorption, contribute to the peripheral expansion of the osseous pieces by progressively externalizing their characteristic external features.

And in the area of the alveolar bone, this osteogenic periosteal layer extends and then forms the peripheral layer of the periodontium (ligament) that surrounds each tooth. All of these membranous structures (Fig. 2), therefore have in common the capacity to respond by adaptive remodeling to the stresses produced by their environment. For the child, even if the possibilities of orthodontic dental displacements are well known, the responses to osseous growth can also be obtained when adequate orthopedic forces, of an adequate intensity and direction are applied to stimulate, to slow down, or to redirect growth in some sutural sites.

If the sutures eventually become inactive, these potentialities persist in the periosteal area and throughout the 


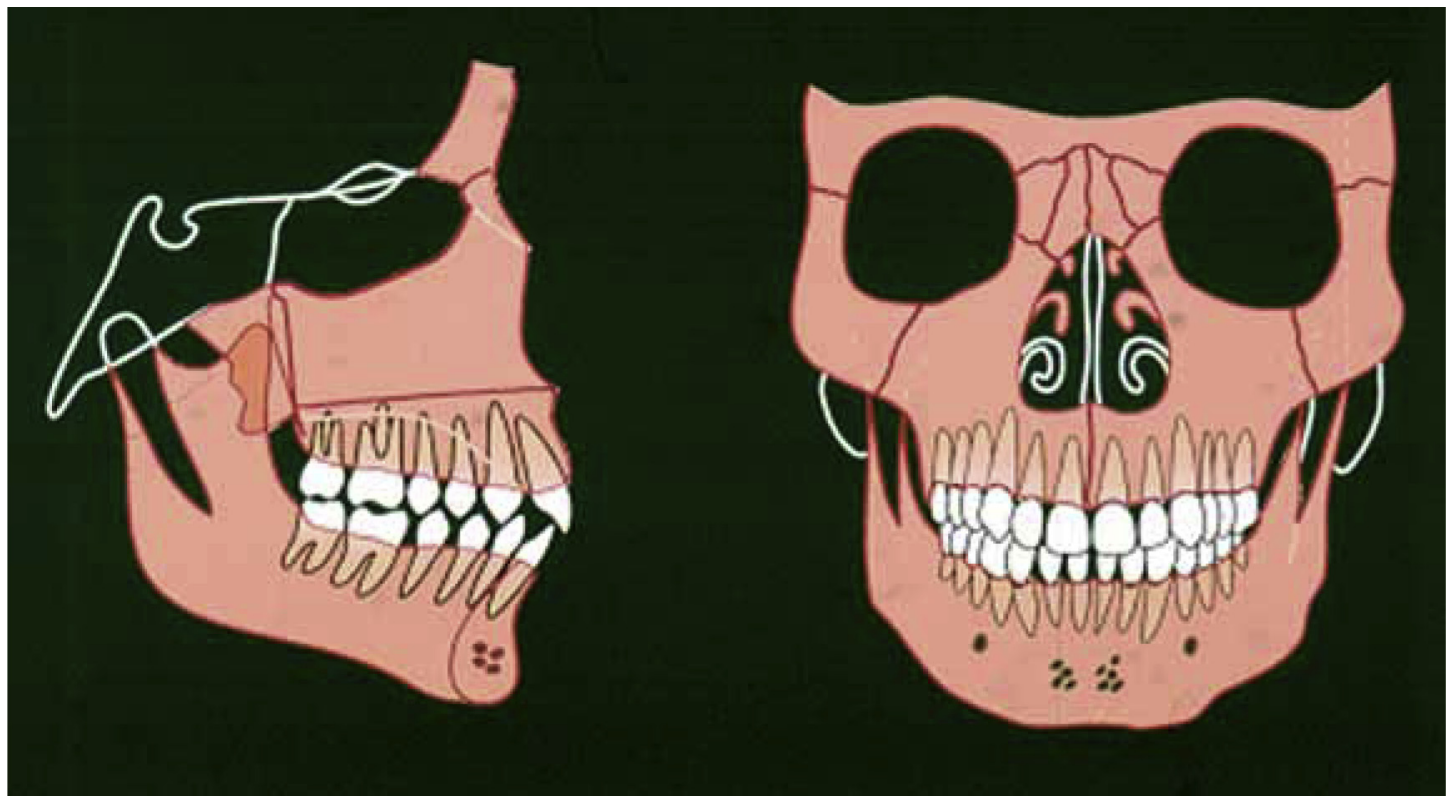

Figure 2

In pink, the structures of membranous origin: most of the bone of the face, the sutures, the periosteum and the ligaments.

periodontium, thereby explaining why orthodontic movements are still possible in adults.

As for the mandible, it also develops, for the most part as the result of a process of membranous ossification (Fig. 2). Around the 40th day in utero, there appears in the mesenchyme, outside of Meckel's cartilage (which goes on to disappear) a point of ossification located just behind the future mental trough. This kernel develops forwards, to the rear and below to become a bony blade that, in angling in the inferior part, will form a bony gutter, the future rough outline of the mandibular body. The two horizontal branches will fuse at the level of the symphysis at the end of the first year of life.

On the other hand, towards the 4th month of intra-uterine life, two secondary cartilages will appear in the same area of the future ascending ramus of the mandible: the coronoid cartilage that will have only a brief existence since, before birth, it is already completely replaced by bone, and the condylar cartilage, which will remain active until after puberty. It therefore will progressively govern the process of endochondral ossification beginning at it origin, behind the future spine of Spix, but will continue, throughout the entire postnatal growth period, to elongate upwards and backwards in order to participate in the building of the ramus and more particularly of the condylar apophysis (Fig. 3). This cartilage is endowed, by its very nature, with a potential for hereditary growth inherent in all cartilage, but has, in addition, adaptive growth potential regulated mainly 


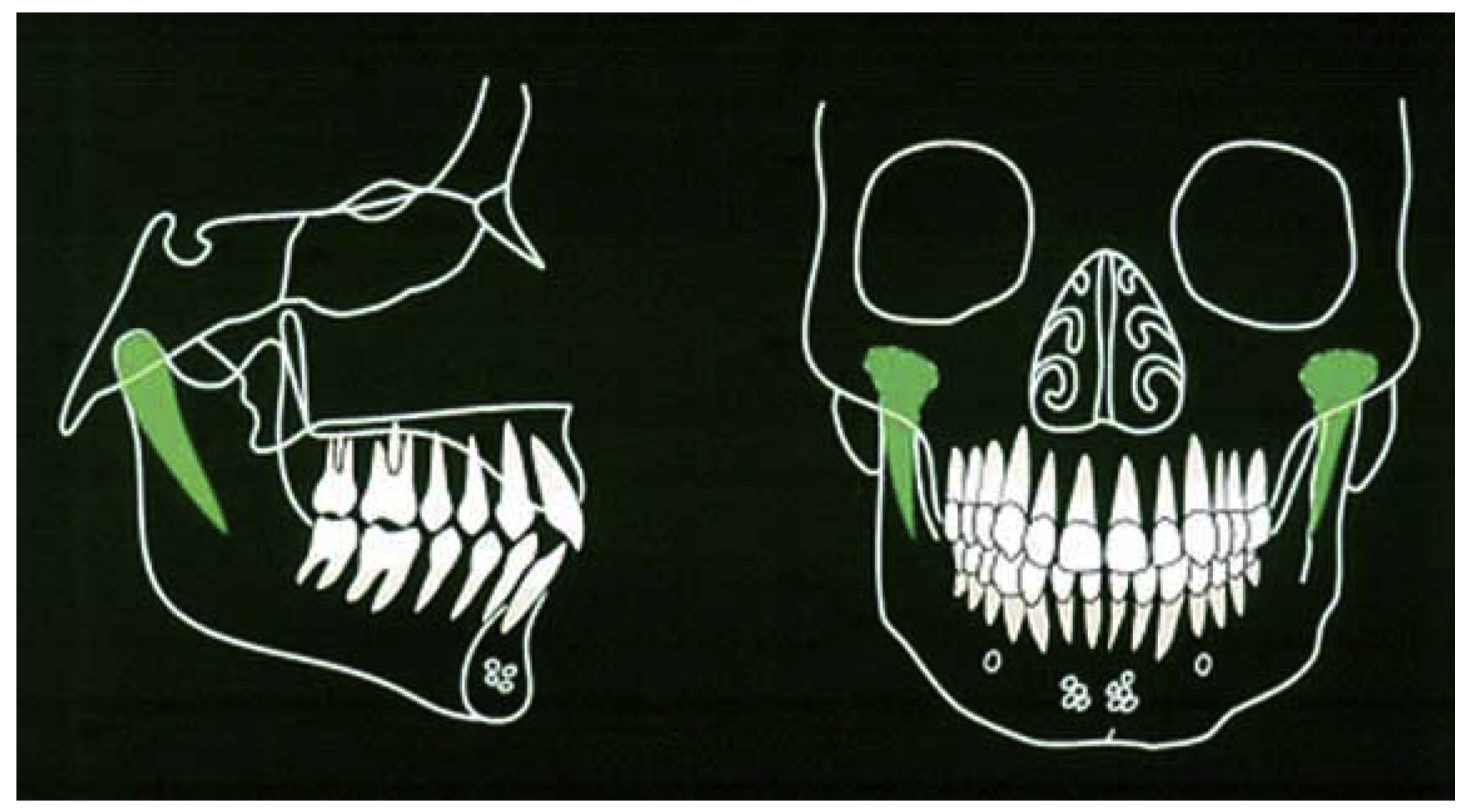

Figure 3

In green: the condylar cartilage and its endochondral ossification.

by activities of mandibular propulsion bringing into play the external pterygoid muscles as well as other muscles.

This capacity therefore provides the condylar cartilage with a secondary mode of growth, in addition to its primary autonomous potential, conditioned by the functional influences of the environment that is similar to the adaptive response of the sutures.

Thus, the mandible, a unique and mobile bone piece, has no postnatal growth sutures with any other bones, but has, besides its potential for periosteal remodeling, a process of adaptive growth, that is furthermore largely stimulated in the presence of growth hormones. Moreover, the abundance of muscular insertions that cover the mandibular surfaces, show the multiple functional stresses that can stimulate the process of remodeling and growth.
As a consequence, most of the growth of facial bones, in both the maxilla and the mandible, is certainly dependent on constitutional genetic factors for the placement of the structures and their direction of growth as well as their individual potential for growth and the capacities for biological responses of the different tissues, that largely depends, for their growth in size and in position, on numerous environmental factors, which modulate this potential. Some of these factors are praxic and orofacial tonic activities (feeding, chewing, swallowing, breathing, postural tonus ...) and their dysfunctions.

These considerations therefore help us understand that in early childhood, dento-maxillary deformations may progressively appear, take shape and even worsen in a functional environment which produces insufficient, exaggerated or disruptive stimulation 
even though there was no hereditary predisposition.

Artificial feeding with a bottle, the absence of mandibular propulsion (functional prehensile-bite) and/or insufficient chewing efficacy - often while eating tenderized and soft foods - deprive the process of adaptive growth of crucially important stresses.

An atypical or immature swallowing pattern, the persistence of habits such as thumbsucking or predominantly mouth breathing, are also some dysfunctional contexts that can disturb the growth of the jaws and subsequently, the placement of the teeth and their occlusal relationships.

It is therefore both logical and desirable to correct an unfavorable functional environment as soon as possible in order to avoid making the deficits (or the excesses) of growth as well as any existing deformations worse.

The restoration of an optimal functional environment can then allow the growth process to normalize. But often, the deformations already in place can be such that they constrain, even prevent, the return to a normal functional context. In this case, we have to consider orthodontic treatment, that frequently becomes orthopedic, in order to help nature compensate for these growth deficits and restore the masticatory apparatus, as soon as possible, to its morphological and functional normalcy.

This is the objective of early treatment in the mixed dentition, or perhaps even in the deciduous teeth. And it can produce significant results because the jaw structures, due to their embryological origin and their characteristic adaptive tissues, can produce responses when appropriately adapted stimulations are used. Compensatory growth is therefore possible!

Is the growth potential of a young child, well before the start of the prepubertal growth spurt, sufficient to allow for quantitatively adequate responses at this age? In other words, is "compensatory growth sufficient?"

Can we possibly obtain targeted growth responses in a young child?

Many practitioners prefer to wait for the beginning of the pre-pubertal growth spurt to begin treatment until all the permanent teeth are in the mouth but also due to the significant growth potential present at this time. The start of the pre-pubertal growth spurt is clearly evident when you consider the significant increase in height during this period culminating in the adult height. During the growth spike, the increase in height can reach 10 to $12 \mathrm{~cm}$ per year. In girls, the spike begins between the ages of 10 and 11 at least two years earlier than for boys - and is less intense but the subsequent growth is slower since after growing rapidly for 2 to 3 years, the growth of girls ends rather abruptly.

During this period, all the growth sites are active due to the increase in the amount of growth hormone, including the facial structures (suture, cartilage, condylar ... .). It is therefore in fact a very favorable time to achieve 
adaptive remodeling in response to our therapies. But it is also the last time in life that we can expect to see such growth responses. Once the spike is past, the amount of growth diminishes and is gone at the beginning of adulthood. This period therefore represents the last chance to obtain orthopedic corrections for any deformations that are present. When this window of opportunity closes, the discrepancy between maxilla and mandible cannot be corrected except by orthognathic surgery or compensated, in a limited way, by orthodontic treatment for adjusting the relationships between the dentoalveolar processes.

Even if the growth spurt is undeniably important, we have to be very aware that the majority of growth has already been expressed before the pre-pubertal growth spurt. Since birth and for more than a dozen years, growth pursues a rhythm, certainly inferior to that of the growth spurt (from 5 to $6 \mathrm{~cm}$ of growth in height per year) but without ever stopping even if it undergoes some fluctuations and slowdowns, in particular with the approach of the pre-pubertal growth spurt.

Quantitatively, it is during all these years that most of the facial skeletal structure is formed within the functional environment. Little by little, the qualities of this environment externalize the shape and the meshing of the dental arches.

Therefore, it is important to not let this potential for growth slip by that might possibly provide responses to therapeutic stimulations from the earliest years of childhood.

However, the younger the child, the faster the rate of growth is. In particular, at birth, it is considerable because it follows the intra-uterine growth where, in the space of 9 months, we witness a growth in stature of around $50 \mathrm{~cm}$ ! Never again will a child know such a growth potential. That is why, during the first year of life, prolonged maternal breastfeeding is a powerful engine for the development of the jaws, especially the mandible.

If after the eruption of the deciduous teeth, at around age 3, we notice that a malocclusion or a deformation appears, then it is already time to intervene to treat the dysfunctional etiologies and compensate for the deficits of growth that are induced in order to better prepare the mouth for the eruption of the permanent teeth.

All problems cannot be treated or avoided, in particular those that occur when the permanent teeth erupt, but the normalizations that have already been achieved can only help in the ultimate development of a more harmonious masticatory apparatus.

In many cases, based on the phenomena of development related to the dentition, a second phase of treatment will become necessary in order to completely straighten the permanent teeth and to ensure a correct occlusion. But then this later treatment is often simpler, shorter and requires fewer extractions of permanent teeth.

If normalization is achieved at a young age, will it maintain itself or on the contrary will it lead to relapse since it was obtained too soon before the complete permanent teeth? In other words, "is the compensatory growth stable?"

The stability of the corrections obtained during early treatment is often 
considered to be more risky. First of all, we can see signs of a tendency to relapse after early treatment, as we can see it after later treatment. It is often in situations where the teeth have been moved into positions that are biomechanically unstable due to their inclination or their position in relation to the supporting bone, depending on the resultant functional postural and praxic activities that apply pressure on the dental corridor. Currently, many relapses particularly of the incisors, are prevented or masked by the placement of permanent retention bonded to the permanent teeth, that cannot be considered for young children, in the deciduous or mixed dentition.

In addition, at the end of later treatment, the growth potential is almost exhausted although the remodeling activities continue to persist, but at a very weak rate throughout life. This certainly contributes to stabilize a good number of the acquired results, as it surely explains why a good number of malocclusions and deformations, some of them severe and non-treated, do not worsen to any extent over the short term.

But in the young child, the dentoalveolar structures that have been normalized by early treatment, will, once the appliance is removed, be confronted anew, not only by a functional environment but also by a potential and perhaps an individual growth pattern that will continue to push out throughout the years.

If the hereditary or congenital growth pattern is unfavorable from the start, relapse is often frequent. This is the case in chromosomal anomalies, some congenital maxillo-facial malformations, and innate neuro-muscular deficiencies or in certain hereditary deformations.

But most often the relapse of the results obtained by early treatment stems from the fact that a normalized growth pattern had not been accomplished at the end of treatment, in other words the normal stimulations of growth had not been re-established. The child resumes or continues his either inadequate or disturbed anterior mode of growth and the formerly corrected deformation reappears. The cause of this negative development usually occurs because the disrupted functions, which originally caused the deformation, have not been corrected and the original dysfunctional environment continues to provide inadequate stimulations.

Therefore, the early maxillary transverse expression in the child who is a habitual mouth breather is indicated and necessary, but the correction will only remain stable over time if it is accompanied by a return to spontaneous nasal breathing and a normalization of the oral, postural and praxic dysfunctions, that were associated with it.

Likewise, the correction of a protrusion or of an incisive (anterior) open bite in a thumb sucker will be stable only if this parafunction is definitively eliminated and if the tongue does not continue to be interposed between the teeth.

This often requires a multidisciplinary causative and therapeutic approach, because obtaining a correction that is solely morphologic by mechanical means alone will not be sufficient to guarantee the result. Besides, it sometimes happens, in young children, that by just correcting the 
dysfunctional context, or simply the physiological process of maturation of praxia, leads to a reduction of the deformations and sometimes to their elimination because the growth processes have spontaneously gotten back on track without any appliances.

Spontaneous corrections of deformations, sometimes observed after cessations of a sucking habit, grinding of the teeth, unlocking the occlusion or maturation of swallowing and of phonation, are examples clearly indicative, on one hand, of the marvelous process of growth compensation that growing tissues at this age can display, and, on the other hand, of the importance of re-establishing an optimal functional environment in order to ensure a normal and stable conformation of the dental arches.

\section{Can we possibly hope that very young child will accept and sufficiently comply with the demands of wearing orthodontic appliances?}

Many parents, as well as practitioners, fear having to begin treatment for young children because they are apprehensive about a lack of cooperation on part of these young patients.

This can be true for the ones younger than 4, but sometimes also for older children. It is often a lack of psychoaffective maturation that shows up in their behavior in the form of reactions that the practitioner (and often the parents!) cannot control.

But in most cases, and in all cases after a child turns 4, it is often possible to have perfect collaboration, as long as the family environment is favorable and you spend time to explain to them how they have to participate during treatment in positive language they understand.

The real problem is that we have to take the plunge and begin early treatment, especially when we haven't had any practice or experience and very often we're not really convinced of how effective or useful they are.

However, with a little hindsight, we can see that when these treatments are well accepted and followed, they fit perfectly into the life of the child and can often be a source of pride for them.

In conclusion, therefore, everything we just stated argues in favor of beginning treatment early, but there are several opposing arguments, in particular, the frequent necessity for a second phase of treatment in the permanent teeth in order to perfect the alignment of the dental arches and to create adequate occlusion. Some then criticize the total duration of treatment, and drawn-out periods of appliance wear and others the fact that treatment in two phases does not produce significant differences in results from treatment done later in one stage in the permanent dentition. In a study done by C. Tulloch, W.R. Profitt and C. Phillips ${ }^{13}$, they compared the results of severe Class II (overjet $>7 \mathrm{~mm}$ ) treated in one later phase (in adolescence), in two phases, where the first is qualified as "early treatment" and consisted in the wearing of extra-oral force or a functional appliance. The results suggest that the two-phase treatments are not more effective than the treatments done in one later phase. However, when considering the age of the children in the "two phase" group one appreciates that the term "early treatment" is applied in fact to 
pre-adolescent children, which is very different from the concept of early treatment from the age of 4 or 5 years, that is proposed in this article!

Finally, the real sticking points are that, on one hand, many of our techniques, and in particular, the fixed multibanded, are inadequate for early treatment in the deciduous or mixed

\section{EARLY MANAGEMENT}

First of all, we might ask how soon can early intervention treatment take place. I think that treatment, depending on the case, can often be started as early as age 4 and for all cases before the age of 7 or 8 years, because after that, it is no longer strictly speaking an early interceptive treatment.

We might also consider what cases require early intervention and what procedures should be used for these interceptive treatments.

There is first of all a series of cases of children presenting with congenital or hereditary deformations that are associated with a syndrome of a malformation of the face such as alveolar and/or palatal clefts, Apert or Crouzon Syndromes, oto-mandibular syndromes, cranio-stenoses ... In all these cases, they are often brought for early intervention in a context that is often difficult due to the severity of the presenting morphological anomalies. The treatment is therefore limited at this age and often consists of trying to compensate for the functional deficits (example: obturator plates in cases of palatal clefts) or of trying the first stage of correction of the deformation. However, given the cause of these anomalies, the only way we can hope dentition, and that, on the other hand, many of our practitioners do not have any practical experience using them, and furthermore, do not usually treat young patients. Therefore, the real problem is that we have to convince practitioners to challenge themselves by changing their usual practice and mindset!

to achieve an early normalization is by eliminating the causes that are responsible for the deformations, but, unfortunately, it is not possible to access the initial cause when it is genetic.

But aside from these sad and fortunately relatively infrequent cases, there are a large number of morphologic anomalies in young children that are secondary to the presence of local oral functional problems. The table on the following page attempts to present a synthesis of the causative functional factors and the multiplicity of their repercussions on the appearance of different deformations in the jaws and the dental arches. Thus, it can be seen that the same deformation can result from functionally different disturbances but also that a particular functional problem can have different repercussions and lead to different morphological deformations.

In Germany, Grabowski and his collaborators ${ }^{5,6,12}$ published several articles in 2007 reporting the results of a large study on young children before assessing the epidemiology of malocclusions and dental malpositions, together with oro-facial myofunctional troubles that were present in the same subjects. In this study, they examined 
766 children in the full deciduous dentition and 2,275 children in the early mixed dentition, that is, older than 8 years of age. They found that normal occlusal relations were present in only $25.3 \%$ of children in the deciduous dentition and that this frequency fell to $7.3 \%$ in the early mixed dentition. Among these problems, they had identified a number of myofunctional, static or praxic problems that increased from the period of the deciduous dentition to the period of the mixed dentition. In fact, the absence of oro-facial dysfunction was present in only $11.2 \%$ of children in the complete deciduous dentition and in $10.2 \%$ of children in the early mixed dentition. They were able to show the relationship between the presence of these functional problems and the appearance and the aggravation of the morphology of the dental arches. They concluded the most urgent need is for preventive and interceptive management that must necessarily include a rehabilitation of the functional environment, in order to reduce the harmful influence, or even to eliminate the causative factors responsible for the malocclusions and the malpositions that are found in young children.

From this perspective, a pragmatic view should strengthen our resolve to intervene both morphologically with early orthodontic, or even orthopedic treatment but also preventive treatments in order to correct dysfunctions and restore normal functions. How should we approach these treatments? Should we first start by normalizing morphology or on the contrary by eliminating functional disturbances? Depending on the existing condition and on the level of cooperation of the child, it seems to me to be more
Non physiologic feeding

Insufficient and/or asymmetric mastication

Thumb sucking

Atypical swallowing

Short lingual frenum ("ankyloglossia")

Low tongue posture

Mouth breathing

Labial, cheek hypo-tonicity/hyper-tonicity

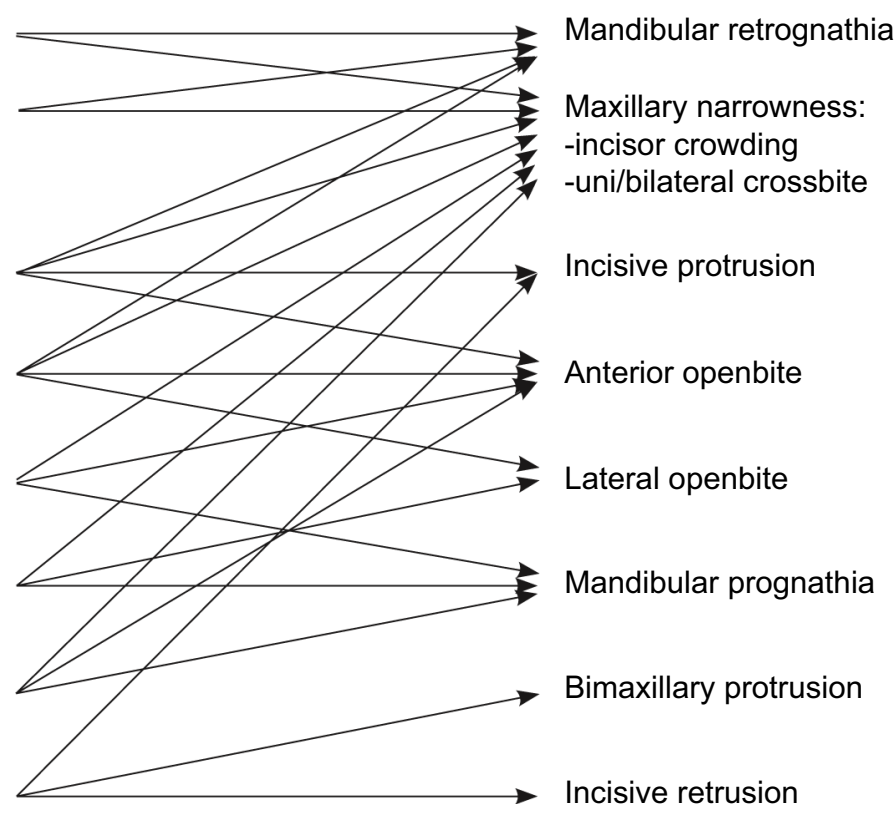

Table

Table presenting a synthesis of the implications of the different functional problems in the genesis of dento-facial deformations. 
logical to start by treating the causes, in other words by treating the dysfunctions in order to contribute to the correction of the deformations and above all to ensure the stability of the results that are achieved. However, in some cases, the morphologic anomaly can already be so severe and the discrepancy between the dental arches so significant, that it is then absolutely necessary to begin by normalizing the relationship between the arches, in order to secondarily make functional reeducation possible, then the morphologic environment can allow the organs (tongue, lips) and the functions (mastication, deglutition, breathing, posture), to recover a static and praxic equilibrium, with retraining exercises that are now possible and effective due to the normalization of the morphologic structures.

\section{Early orthodontic (or orthopedic) treatment}

As shown in the table that synthesizes the etiologic contexts and their orthodontic consequences, there are multiple deformations that can already appear in a young child who is in the deciduous dentition. Among the conditions that are found, various appliances can be suggested to prevent these malocclusions from worsening and to start correcting the morphological anomalies that are already in place.

Various devices can be used, as an adjunct to functional re-education, to facilitate, with accurate proprioceptive feedback, the creation of a new engram of preferred postural and praxic patterns to ensure the development and the recovery of the optimal stres- ses in the functional environment. Some devices that are apparently passive such as buccal screens, myofunctional trainers as well as functional re-trainers, represent simple but often effective ways to help in the correction of functional problems (sucking, interposition of the tongue, atypical swallowing, relaxed posture "half open mouth" ...) and of the most physiological possible maturation of the affected speech, allowing for as well, in many cases, "spontaneous" correction of some dental malpositions or malocclusions (anterior or lateral open bite, incisor protrusion, maxillary narrowness ...). These systems of screens, by blocking the tongue or lip dysfunctions from affecting the dental corridors, therefore promote not only their favorable development towards an apparent "self-correction," but also contribute, over time, to ensure the normal development of the dental arches and to prevent newly erupted teeth from being adversely affected. Therefore, they certainly provide an invaluable contribution to interceptive orthodontics, but also a useful, if not necessary, adjunct, to aid in the therapy of retraining the altered functional patterns.

Additionally, in modern society, young children (and older children, as well as many adults!) present an inadequate masticatory function, a sort of masticatory "impotence" that deprives the jaws of many functional stimulations, that are nevertheless necessary for their harmonious growth. As this has already been mentioned previously (Limme ${ }^{7-9}$ ), this apparently harmless, but in reality deplorable condition, results from profound changes that appeared, over time, in the consistency and the hardness of food in our modern diet, that provides less and 
less stress in the work of chewing. Thus, instead of presenting a progressive wearing of the deciduous dentition (attritional occlusion), that might indicate the quantity and the ease of movements linked to chewing, for the most part, our children maintain a deciduous dentition with completely intact cusps, responsible for a type of occlusal locking, thus preventing a harmonious development of the arches in a transverse direction as well as sagittal growth of the mandible. Selective occlusal grinding are therefore often strongly recommended to bring the deciduous dentition into this stage of maturation that make the expression of the growth processes possible. We have to be particularly attentive, as previously mentioned $\left(\right.$ Limme $\left.^{8}\right)$, to scrupulously respect the symmetry of movements and to restore alternating habitual unilateral chewing. Furthermore, in most children who already present with a significant deficit of a physiological transverse expansion of the arches, the restoration of physiological mastication is no longer adequate so as to allow for morphological recovery with regard to the amount of growth. It is then often advisable to use functional appliances for transverse expansion, such as flat plane occlusal rails as described by P. Planas ${ }^{11}$, in order to free up the existing intercuspation and to foster spontaneous mandibular excursive movements in various directions. These appliances, that can be thought of as "gymnastic" appliances for the masticator muscles, can be very useful in young children to stress the physiological processes of transverse growth of the dental arches and thus relieve in an interceptive or preventive fashion, signs of anterior crowding, that appear as the permanent incisors erupt between 6 to 8 years. The clinical results showed that the transverse expansion obtained was significantly greater than the simple intercanine growth that was normally expected, allowing for a preventive solution of cases of severe crowding that later might have led to the decision to extract permanent teeth. Unfortunately, these appliances are poorly understood in the profession and in the scientific literature, that doesn't have a single evidence-based article attesting to their merits. This therapy, that Planas calls "neuro-occlusal rehabilitation" deserves wider exposure and its implementation should be promoted among concerned practitioners who are considering early interceptive treatment. Unfortunately, we have a real problem due to a lack of training and to the challenging task of changing the mindset of practitioners, who refused, at least at some point, to move in this direction!

Maxillary transverse arch expansion appliances are undoubtedly better known. In connection with the point previously mentioned, Th. De Coster ${ }^{1,2}$ compared two groups of children in regard to the need for premolar extractions: in the first group of 146 children, early maxillary expansion in the mixed dentition reduced the extraction of permanent teeth to $1.35 \%$, while in a group of 347 children, who did not have the benefit of this first phase of early expansion treatment, $10.33 \%$ of them had to have extractions of permanent premolars. The interceptive phase thus significantly reduced the need to extract premolars later.

Other sectorial removable expansion appliances can also be considered for very early use in the deciduous teeth. 
These devices are used, as shown by M.J. Deshayes ${ }^{4}$, to provide for localized stresses in particular in the maxillary arch presenting obvious signs of transverse asymmetry (by a lateral under-development of a sector) or by a sagittal asymmetry (by mesialization of one side in relationship to the other). The purpose of these appliances is to reestablish the harmony of shape and symmetry of the dental arch by soliciting the asymmetric growth activities in cases where the growth of some areas at the same level as the cranial base was disturbed or blocked due to biomechanical barriers related to osteopathic lesions. After the relationship between the dental arches is normal and symmetrical, it is possible to restore a harmonious growth pattern and block the progression of the deformation that was developing. However, this interesting method of management requires knowledge and appropriate training.

Finally, in the most severe cases, where there are more extensive deformations that attest to profound functional problems or to primary anomalies of development, there may be indications, even in the deciduous teeth, for considering interceptive treatment involving the use of orthopedic appliances, such as appliances to expand the mid-palatal suture or of facial masks for maxillary protraction. In this case, the patients are often children who present with a severe dysfunctional environment, associated with habitual mouth breathing, a juvenile swallowing pattern and a tongue that is kept low in the mouth. Unilateral or bilateral or anterior crossbites when present in children, require, in addition to a vitally important functional management plan, aggressive treatment of the basal structures, in order to restore a normal growth pattern as fast as possible and therefore avoid aggravating the existing problems. The disjunction of the midpalatal suture can thus rapidly bring about, not only correction of the transverse crossbites, but also the restoration of the volume for the tongue space, therefore allowing it to rest in place correctly at the level of the palate, and as well, to obtain, in the nose an increase of the volume of the nasal fossae and therefore their permeability. In addition, the significant expansion that is obtained can provide a quantity of room that is then available for the eruption of the permanent teeth and thus can contribute, in most cases, to the reduction of the need to remove permanent teeth later on. As for the facial protraction mask, it makes it possible to achieve, in cases where severe underdevelopment of the maxilla is responsible for a Class III, a growth response of the maxilla in a forward direction, particularly in young children for whom the sutural activity and the growth potential are still very active.

All these early interceptive treatments that aim to correct the morphology and the relationships of the dental arches are utilized but, as was described previously, it is absolutely necessary to associate them with a correction and a rehabilitation of the postural and speech of the oral region.

\section{Myofunctional re-education}

It is often said that a re-education of the oral dysfunctions and the suppression of parafunctions are indispensible in the scope of our orthodontic treatments. But unfortunately, it must be said that functional management, even 
if is recommended by various practitioners, is often minimally effective and frequently ineffective in obtaining a genuine correction of the affected functional problems. In several countries, many children consult "logopedists," as they are called in Belgium, or "orthophonists," the term used in France or in Canada, or also "logopedists", as they are called in Switzerland. In fact, these practitioners are established and they are involved essentially with the problems of articulation, of voice, of speech and/or oral and written language, namely, with problems concerned essentially with communication. Abnormalities include dyslexias, dysorthographias, dysarticulations, retardations of language and speech, dysphasias ... Their training also makes them competent to deal with issues in swallowing and in bucco-facial motor movements. Unfortunately, experience shows that treatment for speech or language therapy takes place most often in a school setting, where the objective is to effect some improvement, or even completely correct the disorder, as it pertains to oral and/or written language, which in itself is quite commendable and often necessary in order to improve the performance of the child and to give him a sense of confidence. Most speech pathologists are often working exclusively on remediating problems of articulation and language but when an orthodontist refers a child to them who presents with more serious dysfunctions (mouth breathing, posture, atypical swallowing ...), it turns out that the management may be inappropriate and not very effective. Often after many re-training sessions, there is little objective change in the postural comportment and oro-buccal praxic speech and various studies report a large percentage of failures to re-train by speech therapists, as high as 80\% in the most complex cases. As a consequence, many children, who need intensive treatment for functional problems that are responsible for deformations, are not reliably rehabilitated and continue to present with imbalances that had predisposed them to the development and persistence of their malocclusions and dental malpositions. Is it possible that these retraining therapies are really just ineffective and useless?

In order to answer this question, we need to be aware that there is, in a variety of postural and praxic patterns, an inherited hierarchy of the chronology of the sensory-motor development and of the physiological process of maturation along with the psycho- or tonico-emotional restrictions. These corrections require some modifications such as relearning and re-training of abnormal behavior that had been poorly ingrained and that now must follow a progressive and structured program while respecting the priorities in the order of the newly acquired behaviors that we wish to promote. Therefore, wanting, as is too often the case, to work immediately on the retraining of articulation and on problems of language, without having achieved beforehand the restoration of normal oral breathing, normal resting position of the tongue, normal mature correct swallowing, can only lead to failure if the primary functions not have been rehabilitated. According to G. MartinotRandoux ${ }^{10}$, the management must begin with a treatment team discussion about the functional origin of the persistent deformations and with a precise analysis of the action of the 
muscular force that fosters these dysfunctions or hinders the effects of the orthodontic treatment.

Then, prioritizing the objectives of speech therapy management implies:

- first of all, an awareness of the body supporting element that will secondarily determine the resting position, the static body position and subsequently, the muscular equilibrium that is indispensible for the development of basic primary functions. Exercises on a carpeted floor, that bring into focus the perception of the body and of its spatial orientation and postural behavior, which are frequently inadequate, will allow for the reestablishment of equilibrium of the muscle chains to retune the body and to facilitate the discovery of a new craniocervico-facial equilibrium;

- then, the restoration of habitual nasal breathing, including throughout the night. Natural and spontaneous maintenance of lip closure is also done prior to the chronological sequence of re-training. This implies that the nasal fossae must be permeable (or made permeable), or enlarged (cf. disjunction) and that breathing function must be revisited in the overall context of the muscular contractions that comprise this function. The return to exclusive and natural nasal breathing is an indispensable preliminary step for obtaining the other corrections;

- if these first steps are accomplished, the tongue resting position will be almost produced naturally; it's just a matter of first consciously positioning the tongue that will then become natural;
- the re-training of swallowing and therefore the reprogramming of the stages of its physiologic maturation, come into play next and require the use of discovery exercises, to then reestablish function before the newly corrected praxia becomes natural. The more spontaneous the swallowing function occurs, the better the praxic recovery;

- in some cases, the movement of the masseters must be relearned, re-trained and recommended eating habits must be provided to the parents in great detail in order to help their children eat hard foods (bread crusts, apples, un-cut meats ...) and to encourage them to chew. In parallel, orthodontic treatment with occlusal attritional grinding appliances and, for example, a Planas occlusal rail appliance, can be used to unlock the occlusion and to facilitate mandibular excursive movements;

- finally, when all the primary but essential functions have been retrained, wed can then consider, the cherry on the cake (!) correcting articulation disorders and often we're surprised to see that the correction of phonation is easy and quick, and sometimes even spontaneous, because all the organs and the primary functions, as a result of this hierarchical re-training, have been prepared for the final physiological acquisition that is language.

In addition to this prioritization in the chronology of management, it is critical to adopt a plan of re-training that will not wind up, as is too often the case, by imposing muscular restrictions on a 
child in order to force the child to position his organs the way we want, instead of having the child discover the sensations, and use the input from his proprioception, to achieve, in response to these discoveries, a progressive adaptation and thus a normalization of speech. The principal of re-training is primarily to solicit the "ports of entry" - the proprioceptive efferences rather than to force the child into muscle exercises - motor efferences - in order to obtain a change and above all a new programming of a functional pattern. In other words, re-training must "copy" nature by respecting the link between the acquisition of the gnosis and the secondary development of speech. We need to first reeducate the gnosis (that is to say the structural motor activities coordinated in response to information recognized by the gnosis). Therefore, in a newborn, the function of suckingswallowing is present. It is structured and has been rehearsed during the last months of pregnancy. At that age, it is called infantile swallowing with total interposition of the tongue, given that the tongue is in contact with the lips. This mode of functioning and the position of the tongue, as well as the labio-lingual contacts, are considered to be physiologic at that age. During the course of development, in particular with successive eruption of the deciduous teeth, the oral environment changes, as perceived by the tongue as a result of its sensitive carpet covering, that is exceptionally rich in terms of the density of its neuro-sensory receptors. Proprioceptive sensations that result modify the primary gnosis and engender modification in the praxic behavior of the tongue, that must adapt itself to the new environment: the tongue will progressively cease to be interposed and to be in contact with the lips. These are the first steps of infantile swallowing as it matures towards adult swallowing. These changes begin to be effective normally around age 4 , the period of time when the masticatory function begins to become increasingly more structured. A thoroughly understood and effective re-training is based on these natural processes of development and maturation, if the practitioner really wants to achieve a true physiologic re-programming of normal speech. It is therefore useless to force a child to wear a lingual appliance over the palatal papilla if, for example, sucking is still present and if the sensations have not been adequately retrained in order to allow him to progress towards the target maturation. The contact of the tongue-lips is present and normal at the beginning of life. The child wants this contact and it remains as parafunctions. It is therefore necessary to disconnect the child from this perception in order to allow him to discover his intra-oral environment and thus to bring about postural and praxic modifications in the behavior of the tongue. This is why the different systems of buccal screens can be help the child in the discovery of new sensations and of new points of support. Therefore, we may also spontaneously observe the movements induced from the elevation of the dome of the tongue against the palatal vault, as pointed out by P. Fellus ${ }^{3}$. But this also requires that the base of the tongue be stabilized before the movement of the elevation of the back of the tongue can be done, that must be preceded by the closure of the mouth, but especially by a stabilization of the mandible by putting the teeth into occlusion. 
It is perfectly understandable that relearning will be facilitated and is mostly possible at a young age, when the child is still in a learning period, where his neural circuits are still malleable and educable but, will become increasingly problematic later on, when it will be largely past the period of the maturation of swallowing.

This type of myofunctional reeducation therefore clearly exceeds the scope of a simple re-training of articulation and phonation. Experience shows that this re-education requires speech therapists or logopeds with specialized training who have acquired specific expertise in this area. There are also kinesiotherapists who have acquired these skills and who can bring their contributions to re-training, because they are perhaps more used to managing the first steps of the re-training, namely - general posture, static rest and the restoration of effective nasal breathing. In this domain, the use of an osteopath can also be useful if

\section{CONCLUSION}

We are increasingly aware that early treatments are important for normalizing form and function as soon as possible and therefore putting the growth process on the right path for the future. Thus, these treatments, when they are well conducted, can make it possible for the dental arches to develop more harmoniously, even to the point when we'll be able to avoid, in many cases, resorting to the extraction of permanent teeth, a decision that will always be a defacing and regrettable solution. But we have to be very aware that interceptive management not necessary, in particular in cases where the postural maintenance is profoundly altered. Using exercises and manipulations, they can unravel the muscular chains, resolve the muscular tensions and thus facilitate movements.

Clinical experience of the last years shows that this approach to functional re-training can produce spectacular results, to such an extent that, in some cases, the interceptive part of orthodontic treatment can almost prove to be unnecessary. Correction of anterior open bites, or even of lateral occlusal crossbites can simply occur following the normalization of a functional context. These clinical cases demonstrate not only the effectiveness of these methods of re-training but also their necessity in the arsenal of our preventive and interceptive treatments. In summary, the objective of these treatments is therefore to treat dysfunctions in order to correct deformations.

is not a trivial matter and that it increasingly requires a multidisciplinary approach, where not only the dental practitioner or the orthodontist are involved, but also other practitioners such as the ENT physician, speech therapists and experimental speech pathologists, as well as kinesiotherapists and perhaps even osteopaths. This multidisciplinary management undoubtedly represents the future reality of our preventive and interceptive early treatments.

Conflicts of interest: The author declares no conflict of interest. 


\section{BIBLIOGRAPHY}

1. De Coster Th. L'expansion orthopédique précoce du maxillaire : une prévention simple de l'encombrement dentaire. Orthod Fr 1995;66:963-71.

2. De Coster Th. L'expansion précoce du maxillaire. Rev Orthop Dento Faciale 1996;30:469-75.

3. Deffez JP, Fellus P, Gerard Ch. Rééducation de la déglutition salivaire. RueilMalmaison : Éditions CdP, 1995.

4. Deshayes MJ. L'art de traiter avant 6 ans. Éditions Cranexplo, 2006.

5. Grabowski R, Stall F, Gaebel M, Kundt G. Relationship between occlusal findings and orofacial myofunctional status in primary and mixed dentitions. Part I: prevalence of malocclusions. J Orofac Orthop 2007;68:26-37.

6. Grabowski R, Kundt G, Stahl F. Interrelation between occlusal findings and orofacial myofunctional status in primary and mixed dentition. Part III: interrelation between malocclusions and orofacial dysfunctions. J Orofac Orthop 2007;68:462-76.

7. Limme M. Conduites alimentaires et croissance des arcades dentaires. Rev Orthop Dento Faciale 2002;36:289-309.

8. Limme M. L'interception en denture temporaire : mastication et réhabilitation neuroocclusale. Ortho Fr 2006;77:113-135.

9. Limme M. Diversification alimentaire et développement dentaire : importance des habitudes alimentaires des jeunes enfants pour la prévention de dysmorphoses orthodontiques. Archives de Pédiatrie 2010;17:S213-S219.

10. Martinot-Randoux G. Techniques logopédiques de rééducation myofonctionnelle. Thèse de licence en logopédie. Université de Liège, 2008.

11. Planas $P$. Rehabilitation neuro-occlusale $(R N O) 2^{\mathrm{e}}$ ed. Rueil-Malmaison : Éditions CdP, 2006.

12. Stahl F, Grabowski R, Gaebel M, Kundt G. Relationship between occlusal findings and orofacial myofunctional status in primary and mixed dentition. Part II : prevalence of orofacial dysfunctions. J Orofac Orthop 2007;68:74-90.

13. Tulloch C, Proffit WR, Phillips Ceib. Outcomes in a 2-phase randomized clinical trial of early class II treatment. Am J Orthod Dentofacial Orthop 2004;125:657-67. 